190:142-52.

2. Levene RJ, Pollak-Christian E, Wolfram S.A 21st century problem: Cannabis toxicity in a 13-month-old child. J Emerg Med. 2019;56:94-6.

3. Claudet I, Mouvier S, Labadie M, et al. Unintentional cannabis intoxication in toddlers. Pediatrics. 2017;140: e20170017.

4. Thomas AA,Mazor S. Unintentional marijuana exposure pre- senting as altered mental status in the paediatric emergency department: A case series. J Emerg Med. 2017; 53:e119-e123. 5. Moeller KE, Lee KC, Kissack JC. Urine drug screening: Practical guide for clinicians. Mayo Clin Proc. 2008;83:66-76.

6. Davies E, Connolly DJ, Mordekar SR. Encephalopathy in children: An approach to assessment and management. Arch Dis Child. 2012;97:452-8.

\section{Introduction of Proton Beam Therapy in Intracranial Germ Cell Tumors in India}

Intracranial germ cell tumors (ICGCT) represent rare tumors comprising $1-2 \%$ of brain tumors and $<3 \%$ of all neoplasms in children [1]. Optimal management of ICGCT involves multimodal therapy including surgery, radiotherapy (RT) and systemic chemotherapy [2]. Proton beam therapy (PT) has unique features of delivering sharp fall-off of RT dose resulting in significant sparing of normal tissues compared to traditional photon therapy. We describe our initial experience in treatment of these tumors using image-guided intensity modulated proton therapy (IMPT) at our center, the first and only PT facility in South Asia.

An 18-year-old male presented with history of decreased appetite, weight loss, and generalized weakness for six months. Magnetic resonance imaging (MRI) of brain showed lesions in periventricular region and subsequent stereotactic biopsy was suggestive of intracranial germinoma with CD117 and Oct4 positivity. Cerebrospinal fluid (CSF) analysis revealed increased beta human chorionic gonadotrophin $(\beta-\mathrm{HCG})$ and normal alpha fetoprotein (AFP) with no malignant cells. He received four cycles of etoposide and carboplatin according to ACNS0232 protocol [2], following which, his tumor markers normalized and he was subsequently treated with IMPT to a total dose of $40 \mathrm{GyE}$ in 23 fractions $(23.8 \mathrm{GyE}$ in 14 fractions craniospinal irradiation (CSI) and 16.2 GyE in 9 fractions boost to gross residual and pre chemotherapy volumes) [3]. He tolerated PT well and subsequent MRIs done 6 weeks, 6 months and 14 months post-PT did not show any residual disease. His tumor markers and endocrine values were within normal limits with his height relatively stable as he has already achieved his growth spurt. He has resumed his socio-academic activities and is on regular follow-up for the past 20 months.

A 16-year-old boy presented with sudden onset diplopia since five months. MRI showed well defined lobulated mass lesion measuring $2.4 \times 2.1 \times 3.1 \mathrm{~cm}$ in the region of third ventricle. Tumor marker analysis showed elevated serum â-HCG (1626 $\mathrm{mIU} / \mathrm{mL})$ and $\operatorname{AFP}(2.0 \mathrm{ng} / \mathrm{mL})$. CSF analysis reported increased $\beta$-HCG measuring $3589 \mathrm{mIU} / \mathrm{mL}$ and AFP measuring $0.47 \mathrm{ng} / \mathrm{mL}$. A diagnosis of NGGCT (choriocar-cinoma) was made and he subsequently received four cycles of ifosfamide, cyclophosphamide and carboplatin (ICE), followed by IMPT to a total dose of $54 \mathrm{GyE}$ in 30 fractions (CSI 30.6 GyE in 17 fractions followed by boost of $23.4 \mathrm{GyE}$ in 13 fractions) [3].
Follow-up MRI post-PT after 2 month and 18 months showed interval decrease in residual disease. Post-PT tumor markers were normal and endocrine functions optimal, with the patient's height relatively stable. He has been on regular follow-up since past 20 months and has been continuing his normal socioacademic activities.

A 15-year-old female with amenorrhea, presented with increased thirst, micturition, weight loss, and blurring of vision towards left side over a period of two years. Visual perimetry showed bilateral temporal hemianopia. MRI brain with spine screening revealed a $2.1 \times 2.3 \times 2.3 \mathrm{~cm}$ suprasellar lesion compressing the optic chiasm. She underwent a right pterional craniotomy and gross total resection of lesion, reported as intracranial germinoma. Her tumor markers (serum and CSF) showed mild elevation of $\beta$-HCG $(2.8 \mathrm{mIU} / \mathrm{mL})$. She was on thyroid, cortisol and desmopressin supplements post-surgery because of decreased endocrine functions. Her neurocognitive evaluation before proton therapy showed her in the high average range. She received four cycles of three weekly etoposide and carboplatin followed by IMPT (Fig.1) to a total dose of $40 \mathrm{GyE}$ in 25 fractions ( $24 \mathrm{GyE}$ in 15 fractions to whole ventricular volume and $16 \mathrm{GyE}$ in 10 fractions to tumor bed) [4]. Post-PT tumor markers were within normal limits. Follow up MRI after one, six and twelve months did not show any residual disease. Post proton therapy, her endocrine function did not deteriorate and she was continued on hormone supplements. Subsequent ophthalmic evaluation showed no visual deficits. The patient has been on regular follow up for the past 15 months and has resumed her normal academic activities.

For all these patients, cases were discussed in multidisciplinary tumor boards. Patients, after customized immobilization, underwent a planning CT and MRI. Dedicated PT plans were generated for each case using Monte-Carlo optimization and 3-4 PT fields [3]. Treatments were delivered on a daily basis ( 5 fractions a week) after carefully laid out quality assurance checks as per institutional protocols. Significant reduction of the radiation dose to critical structures such as hippocampi and cochlea were observed.

RT is an integral part of treatment of ICGCT but can be associated with considerable late effects including neurocognitive disturbances and risk of secondary cancers, and chemotherapy alone is insufficient due to high rates of local and metastatic recurrence. Current standard of care is ventricular radiotherapy in case of localized and CSI in case of disseminated germinomas [4,5]. In comparison with conventional radiotherapy, PT due to its unique physical and biological 


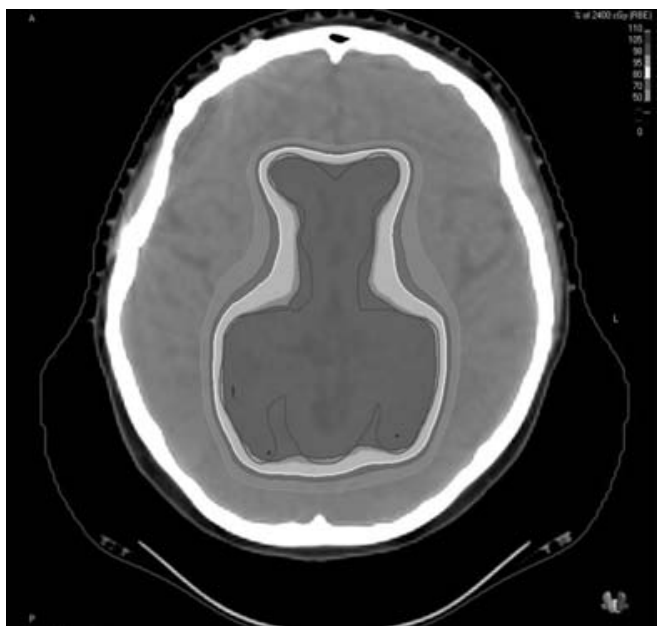

Fig. 1 Dose distribution of whole ventricular radiotherapy using Intensity modulated proton therapy.

characteristics results in delivering low entry dose and deposit the majority of their energy at the end of their path, yielding a typical dose energy peak called 'Bragg peak.' This steep fall-off allows for the delivery of high radiation doses to the tumor and sparing of tissue beyond the tumor. All our patients underwent PT as a part of curative management and tolerated the treatment well. One patient treated with CSI had grade III neutropenia managed conservatively, whereas others did not experience more than grade II toxicities. Mean dose to hippocampus for all our patients was less than $30 \mathrm{~Gy}$, below the accepted threshold for intelligence quotient preservation [6]. All patients could resume their normal schooling after the treatment, with no impact so far in their educational activities, and maintained quality of life. However, neurocognitive assessments were not available for two out of the three patients, and could not be planned due to the logistic challenges because of the ongoing COVID-19 pandemic.
We have successfully implemented PT in the treatment of ICGCT in India. PT should be considered as a treatment option for optimal management of these curable tumors. Further follow up is required to assess the long-term sequelae of treatment in these patients.

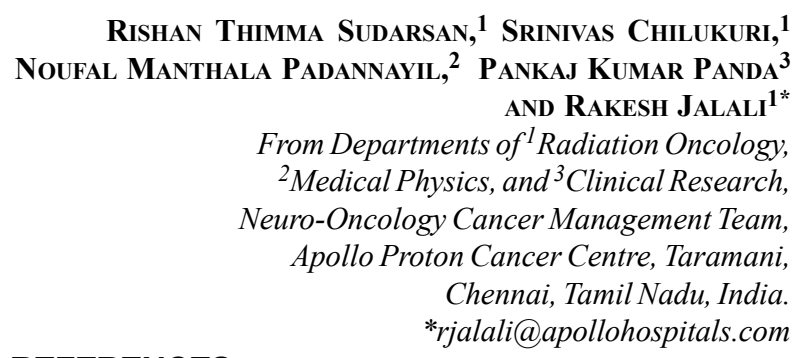

\section{REFERENCES}

1. Kakkar A, Biswas A, Kalyani N, et al. Intracranial germ cell tumors: A multi-institutional experience from three tertiary care centers in India. Childs Nerv Syst. 2016;32:2173-80.

2. Calaminus G, Kortmann R, Worch J, et al. SIOP CNS GCT 96: Final report of outcome of a prospective, multinational nonrandomized trial for children and adults with intracranial germinoma, comparing craniospinal irradiation alone with chemotherapy followed by focal primary site irradiation for patients with localized disease. Neuro Oncol. 2013;15:788-96.

3. Tonse R, Chilikuri S, Shamurailatpam D, Jalali R. Introduction of image-guided pencil beam for skull base tumors in India: A report of two cases and a brief review of the literature. Neurol India. 2020;68:42.

4. MacDonald SM, Trofimov A, Safai S, et al. Proton radiotherapy for pediatric central nervous system germ cell tumors: Early clinical outcomes. Int J Radiat Oncol Biol Phys. 2011;79:121-9.

5. Rogers S, Mosleh-Shirazi M, Saran F. Radiotherapy of localised intracranial germinoma: Time to sever historical ties? Lancet Oncol. 2005;6:509-19.

6. Goda JS, Dutta D, Krishna U, et al. Hippocampal radiotherapy dose constraints for predicting long-term neurocognitive outcomes: Mature data from a prospective trial in young patients with brain tumors. Neuro Oncol. 2020;;22:1677-85.

\section{Acute Meningoencephalitis in a Child Secondary to SARS-CoV-2 Virus}

We report a case of cerebrospinal fluid (CSF)-proven severe acute respiratory syndrome coronavirus-2 (SARS-CoV-2) in a child with acute meningoencephalitis.

An 11-year-old boy presented with one day history of fever, headache, vomiting and altered sensorium. There was no history of cough, fast breathing, rash or abdominal pain. On examination he was hemodynamically stable with a Glasgow coma scale (GCS) of 9 (E3 V2 M4). There was no cranial nerve paresis and he had signs of meningeal irritation (neck stiffness and positive Kernig's sign). In motor functions, he had increased tone with brisk reflexes and extensor planters in both lower limbs. Fundus examination was normal. Child was managed in pediatric intensive care unit as per the standard protocol for acute febrile encephalopathy with empirical broad-spectrum antibiotics and acyclovir along with other supportive care. Blood investigation showed severe lymphopenia (absolute lymphocyte counts $700 / \mathrm{mm}^{3}$ ) and raised inflam-matory markers (C-reactive protein-18 mg/dL, lactate dehydro-genase$4000 \mathrm{U} / \mathrm{L}$, ferritin-2400ng/ml, D-dimer-51091 ng/mL) with deranged liver functions. CSF examination showed pleo-cytosis (75 cells) with lymphocytic predominance $(80 \%)$, very high protein $(696 \mathrm{mg} / \mathrm{dL})$ and normal sugar levels. The RT-PCR test for SARS-CoV-2 was done on a nasopharyngeal swab and CSF because of the outbreak situation and was found to be positive in both. CSF was negative for other neurotropic viruses (herpes, varicella and entero virus). A head contrast enhanced computed tomography (CECT) scan was normal. 